\title{
Balkanologie
}

Balkanologie Revue d'études pluridisciplinaires

Vol. III, n 1 | 1999

Volume III Numéro 1

\section{Staline et les Balkans}

Un document important sur la réunion du 10 février 1948

\section{Vasil Kolarov}

\section{(2) OpenEdition}

\section{Journals}

Édition électronique

URL : https://journals.openedition.org/balkanologie/285

DOI : 10.4000/balkanologie.285

ISSN : 1965-0582

Éditeur

Association française d'études sur les Balkans (Afebalk)

\section{Édition imprimée}

Date de publication : 1 juillet 1999

ISSN : 1279-7952

\section{Référence électronique}

Vasil Kolarov, «Staline et les Balkans », Balkanologie [En ligne], Vol. III, n 1 | 1999, mis en ligne le 03 juin 2008, consulté le 28 juin 2022. URL : http://journals.openedition.org/balkanologie/285 ; DOI : https://doi.org/10.4000/balkanologie.285

Ce document a été généré automatiquement le 17 décembre 2020.

(c) Tous droits réservés 


\title{
Staline et les Balkans
}

\author{
Un document important sur la réunion du 10 février 1948
}

\section{Vasil Kolarov}

\section{NOTE DE L'ÉDITEUR}

\section{Présenté par Bernard Lory}

1 Une illusion splendide et exaltante a régné sur les Balkans entre la fin de la Deuxième Guerre mondiale et le printemps de 1948, celle que l'idéologie communiste pouvait résoudre les problèmes nationaux de la Péninsule. La lecture des processus historiques en termes de lutte des classes, la foi dans l'internationalisme prolétarien et le recours au modèle fédéral, tel que le proposait l'URSS, servaient d'assises à cette grande espérance.

2 Le fédéralisme permettait à la Yougoslavie de redémarrer après une épouvantable guerre civile. La reconnaissance de l'identité nationale macédonienne en Yougoslavie permettait le déblocage d'une région et d'une société toute entière. L'on crut que la même recette pourrait s'appliquer à la Macédoine du Pirin (bulgare) et à la Macédoine de l'Égée (grecque) en oubliant que les contextes socio-politiques y étaient fort différents. On crut pouvoir régler la question albanaise, mal tranchée en 1913.

3 Des jalons politiques furent posés entre la Bulgarie et la Yougoslavie (accords de Bled, $1^{\text {er }}$ août 1947, et d'Evksingrad, 27 novembre 1947) tandis qu'une étroite collaboration politique, économique et militaire se développa entre la Yougoslavie et l'Albanie. Les trois pays faisaient preuve de solidarité envers la lutte armée menée par le KKE en Grèce. L'objectif annoncé était une fédération ou confédération balkanique.

4 Ce projet tomba de lui-même avec la crise du Kominform (28 juin 1948) qui exclut la Yougoslavie du camp socialiste. Dans la genèse de cette crise, la réunion secrète qui se tint à Moscou le 10 février 1948 occupe une place déterminante. Cette réunion n'a longtemps été connue que par le récit qu'en fit Milovan Djilas en 1961, en se fiant exclusivement à sa mémoire car il ne disposait plus, à cette époque, de sa documentation personnelle ${ }^{1}$. Plus récemment, Dobrin Mičev a utilisé les notes 
sténographiques de Trajčo Kostov sur la réunion ${ }^{2}$. Une nouvelle source sur cet événement charnière a été publiée par Veselin Angelov, à savoir les notes prises par Vasil Kolarov, immédiatement après la réunion ${ }^{3}$. Ce document nous a paru mériter d'être mis à la disposition d'un public plus large et nous en proposons la traduction française aux lecteurs de Balkanologie.

Le prétexte à la réunion du 10 février 1948 fut l'interview donné par Georgi Dimitrov le 18 janvier 1948 dans le train qui le ramenait de Bucarest à Giurgiu à plusieurs journalistes étrangers. Il y déclara, entre autres,

la question de la fédération est pour nous une question prématurée. Elle n'est pas à l'ordre du jour à présent et c'est pourquoi cette question n'a pas fait l'objet de débats lors de notre conférence. Quand la question aura mûri, et elle mûrira forcément, alors nos peuples, les peuples des démocraties populaires, de Roumanie, Bulgarie, Yougoslavie, Albanie, Tchécoslovaquie, Pologne, Hongrie et Grèce retenez bien, de Grèce aussi ! - en décideront. Ce seront eux qui décideront de sa nature, fédération ou confédération, de savoir quand et comment elle sera formée. Je peux affirmer que ce qu'accomplissent à présent nos peuples facilite dans une grande mesure la résolution de cette question à l'avenir'.

6 La presse occidentale fit un large écho à cet interview, laissant largement entendre que, par la voix de Dimitrov, c'était Staline qui dévoilait ses intentions à moyen terme. La Pravda du 23 janvier résumait l'interview de Dimitrov sans commentaire.

7 Cependant, le 24 janvier, Staline adressa à Dimitrov un télégramme, déclarant que le projet de large fédération incluant la Grèce était «nuisible, portant tort aux pays de démocratie nouvelle et facilitant la lutte des Anglo-américains contre ces pays $»^{5}$. Le 28 janvier 1948, suite à des courriers de lecteurs, la Pravda publiait une mise au point: l'interview de Dimitrov avait été publié dans la presse internationale et la Pravda ne pouvait le passer sous silence, néanmoins la rédaction du journal estimait «que ces pays n'ont pas besoin d'une fédération ou confédération problématique et inventée, ni d'une union douanière, mais de renforcer et de défendre leur indépendance et souveraineté, par le biais de la mobilisation et de l'organisation de leurs forces populaires et démocratiques internes $»^{6}$.

8 Dès le télégramme de Staline, Dimitrov tenta de se disculper : il envoya une lettre d'explication à Moscou et fit publier par l'agence BTA une déclaration officielle à propos du communiqué de la Pravda, où il déclarait qu'» il considérait la question de la création d'une fédération ou confédération comme prématurée et pas d'actualité » et qu'il n'en avait pas été question avec la délégation roumaine, pas plus que lors de la signature des traités avec la Yougoslavie et l'Albanie ${ }^{7}$.

9 Mais ces justifications ne pouvaient satisfaire Moscou. La Pravda refusa de publier la déclaration de BTA, jugée «absolument pas convaincante». Cette dernière fut cependant publiée dans la presse yougoslave. L'intransigeance soviétique n'avait en réalité guère à voir avec l'interview incriminé. Il s'agissait en fait de la montée de la crise soviéto-yougoslave. Staline avait apparemment décidé de reprendre en main un satellite rétif et il convoqua une réunion à trois. Tito sentit fort bien se profiler l'affrontement et s'inventa une maladie diplomatique. Si la Bulgarie était représentée au plus haut niveau à la réunion secrète du 10 février 1948, avec Georgi Dimitrov, Vasil Kolarov et Trajčo Kostov, la Yougoslavie l'était par Milovan Djilas (déjà en URSS depuis un mois), Edvard Kardelj et Vladimir Bakarić. Du côté soviétique: Staline, Molotov, Jdanov, Malenkov, Suslov et Zorine. On ne comprend bien la situation qu'en se rappelant sans cesse l'absence de Tito à la réunion. Comme l'a fort bien observé Djilas 
par le proverbe balkanique " je te parle, ma fille ; écoute, ma belle-fille !», la semonce adressée par Staline à Dimitrov vise en réalité l'absent. Toute la séance est une leçon de Realpolitik de la part de Staline : primauté de la "grande politique" sur les enjeux locaux, froide appréciation des rapports de force, hégémonie de l'URSS sur les satellites... Le carnet de Vasil Kolarov, conservé aux Archives politiques centrales de Bulgarie (f-147, op. 2, a.e. 62) comprend 49 pages manuscrites. Le texte fourmille d'abréviations, dont la teneur est ici restituée entre parenthèses (il y a quelques rares parenthèses qui sont de la plume de Kolarov). Entre crochets on trouvera les explications liées à l'édition du document ${ }^{9}$.

BL

1/ $\operatorname{Tr}$ (aité) b(ulgaro)-youg(oslave)

2/ Interview (de) G. D(imitrov)

3/ Bulgarie [sic dans l'original, la suite du texte montre qu'il s'agit de l'Albanie]

13 I

14 Rem(arques) russes

Gouv(ernment) sov(iétique) ce gouv(ernment)

(Le) tr(aité) déc(ide)

a/ illimité

$\mathrm{b} /$ entre en vigueur immédiatement

c/ allège [mot illisible] les cercles réact(ionnaires)

(Le) gouv(ernement) sov(iétique) ne peut porter la resp(onsabilité) d'un pacte d'une importance én(orme)

le c/ innaceptable et intolérable

Interview de G. D(imitrov)

Cam(arade) Dim(itrov) se laisse emporter. A exposé un plan [illisible] féd(ération) et conféd(ération) [illisible]. Polit(iquement) nuisible - facilite le combat des Angloamér(icains) contre les dém(ocraties) pop(ulaires). Précipité et irréfléchi. Faute tact(ique) grave. (Les) cam(arades) pol(onais) ont cru d'une partie de l'interv(iew) que c'est le p(oin)t de vue de $\mathrm{M}$ (olotov). Mais quand on les a interrogés sur ce qu'ils pens(aient) en fait, ils ont rép(ondu) : nous ne sommes pas d'accord.

Analogie avec l'union douan(ière) de 1904: "alors les Allemands s'y opposèrent, maintenant ce sont les Russes $»^{10}$.

III

$\mathrm{Qu}($ estion) alb(anaise)

Dans les proch(ains) jours devait passer à l'act(ion) la division youg(oslave) introduite en territ(oire) alb(anais).

Ils n'avaient pas averti $\mathrm{M}$ (olotov). Après question de $\mathrm{M}$ (olotov), Tito a répondu que l'envoi de la div(ision) est suspendu.

Stal(ine) : Je commence à douter de (la) victoire des partisans grec(s). Il y a d'ailleurs un argument pour limiter (les) partisans. Les Américains ont besoin de la Grèce. Il n'est pas prouvé que les $\mathrm{B}$ (alkans) iront plus mal si le gouv(ernement) mon(archo)-fasc(iste) l'emporte en Grèce.

[Jusque là, les notes sont en russe ; la suite du texte est entièrement en bulgare.] 


\section{II.1948}

(Des) diss(ensions) ex(istent) sur trois quest(ions) : le traité bulg(aro)-youg(oslave), l'int(erview) de G. D(imitrov), l'introd(uction) de tr(oupes) yougosl(aves) en Alb(anie).

$1 /$ C'est une erreur que le tr(aité) bulgaro-youg(oslave) ait été conclu, et ce de façon illimitée, avant l'ent(rée) en vigueur du tr(aité) de paix avec la Bulg(arie) ${ }^{11}$.

Pour leur précip(itation), les deux gouv(ernements) ont renforcé les positions des élém(ents) réact(ionnaires) en Angl(eterre) et en Am(érique), en leur donnant prétexte à renforcer (leur) interv(ention) en Grèce contre la B(ulgarie) et la Yougo(slavie). (Le) gouv(ernement) sov(iétique) doit avertir qu'il ne peut prendre la resp(onsabilité) de pactes d'une importance aussi énorme, conclus sans consult(ation) de l'URSS.

Ils étaient d'accord avec nous, mais ils ont agi à l'inverse, sans nous avertir; de telles rel(ations) sont incorrectes et inacceptables.

2/ Ils ont l'impression que le cam(arade) G. D. s'est laissé emporté dans sa conf(érence) de presse et l'interview, en offrant l'opportunité qu'on le provoque sur des affaires dont il ne faut pas parler. Ce n'est ni correct ni souhaitable. L'interview exposait un plan qui va très loin, sans le moind(re) essai de se mettre d'accord avec qui ce soit.

La question est posée d'une féd(ération) ou confédér(ation), d'une union douan(ière), en y incl(uant) et la Pologne et la Grèce. Le cam(arade) G. D. parle de toutes ces aff(aires), sans en avoir été autor(isé) par personne. C'est incorr(ect) sur le fond et nuisible tactiqu(ement) : cela facil(ite) la tâche pour la créat(ion) d'un bloc occid(ental). Pour cela, le cam(arade) G. D. a reçu un avertissement par la voie du parti.

Votre déclaration à la conf(érence) de presse. En ce qui concerne la créat(ion) d'une féd(ération) ou confédér(ation), c'est considéré comme nuisible, cela porte tort aux pays des nouv(elles) démocr(aties) et arrange la posit(ion) des Anglo-amér(icains). Également nuisible et inadmissible est la déclar(ation) sur l'union douan(ière), car on peut y comprendre que s'y associera aussi l'URSS, laquelle aura bientôt un pacte d'assistance mutuelle avec ces pays. On a du mal à comprendre ce qui vous a poussé à faire tant de déclarations à la légère à la conf(érence) de presse.

nous avons discuté avec les camarades polonais, ils nous ont dit : nous avons cru que c'était l'op(inion) de $\mathrm{M}$ (olotov). Tout le monde compr(end) ainsi, que si $\mathrm{D}$ (imitrov) et T(ito) parl(ent) d'une série d'Ét(ats), cela émane de l'URSS. Et, fondamentalement, les cam(arades) pol(onais) ont dit qu'ils étaient contre l'idée de G. D. et la jugeaient incorr(ecte).

28 Nous avons dû prendre position, puisque tous - ennemis et amis - imaginaient que c'était notre p(oin)t de vue. Nous considérons cela (comme) absol(ument) incorr(ect) et inadmissible à l'avenir.

justific(ations) de $\mathrm{D}$ (imitrov) n'ont pas aidé, et ont même compliqué davantage les aff(aires). Il en ressortait, qu'autrefois à l'union douan(ière) fédér(ation) avec la Serbie s'était opposée l'All(emagne) et maintenant l'URSS.

D(imitrov) : Ce n'est pas ce que nous avons pensé.

$\mathrm{S}$ (taline) : Vous êtes un homme pol(itique) et vous devez penser non seulement à vos intent(ions),mais aussi aux conséquences de vos déclar(ations).

Kol(arov) : Nous avons cru que vous étiez même contre l'union douan(ière) entre la $\mathrm{B}$ (ulgarie) et la You(goslavie). 
$\mathrm{S}($ taline) : Nous n'avons pas d'objections à l'union douan(ière) (entre la) Yougosl(avie) et la Bulg(arie), bien au contraire.

$\mathrm{M}$ (olotov) : Le j(ourna)l de Beneš s'est empressé d'écrire « $\mathrm{D}$ (imitrov) a révélé les plans commun(istes), que les com(munistes) tch(èques) répondent à présent $»^{12}$.

D'un autre côté, ces pos(itions) de G. D. sont en contrad(iction) avec la déclar(ation) des 9 partis com(munistes).

S: Votre interview détourne l'att(ention) des questions int(ernes), en disant autre chose que ce qui a été dit à la conf(érence) des 9 partis c(ommunistes) ${ }^{13}$.

$\mathrm{M}$ : Nous considérons tout ceci comme inadm(issible) à l'avenir.

$\mathrm{Z}^{14}$ : Nous avons appris par hasard que, fin janvier, une $\operatorname{div}($ ision) yougos(lave) devait être introduite en Albanie pour la défense conj(ointe) de la frontière. Les Albanais ont dit qu'ils étaient convaincus que cela se faisait avec notre accord.

Il en résulte que nous apprécions différ(emment) la sit(uation) en Alb(anie). On ne peut passer sous sil(ence) une telle diverg(ence). Nous devons disc(uter) ouvertement de ces qu(estions), pour voir comment vous appr(éciez) la sit(uation). À l'avenir, nous consid(érons), que de telles aff(aires) ne doivent pas être tolérées. Nous devons éclaircir les diverg(ences) effect(ives), s'il y a des diff(érences). Les points de vue doivent être exprimés et discutés. Sur de telles questions, il ne faut pas agir sans consultation pour les besoins de la cause et de nos ent(entes) mut(uelles). À l'avenir le cam(arade) G. D. $\mathrm{d}$ (oit) éviter, pour lui-même et pour nous, le danger de telles déclarations.

D: Pour ce qui est de Bled, j'ai reconnu mes torts, pour l'interview aussi. Pour l'augmentation de la puissance écon(omique) et les capac(ités) de déf(ense) des pays, une coopérat(ion) des [texte illisible] pays des nouvelles démocraties nous est indisp(ensable).

S: Vous avez voulu faire un discours novateur, les Pol(onais) et les Tch(èques) se moquent de votre fédér(ation). Demandez-leur s'ils en veulent !

D : C'était nuisible et fondamentalement incorrect. La justification s'adressait à ceux qui auraient voulu utiliser la déclar(ation) contre nous et l'URSS. Je me suis laissé emporter. De telles déclar(ations) ne se répét(eront) pas à l'avenir.

$\mathrm{S}$ : Nous ne nous comprenons pas l'un l'autre. Et vous essayez de le dissimuler.

$\mathrm{M}:$ Ce sont des affaires sérieuses, pas des détails.

S: Vous êtes un homme polit(ique) expérimenté. De quelle erreur peut-on parler (?) L'orientation chez nous est différente, sans que vous-même n'en ayez consc(ience). Il ne faut pas donner d'interviews aussi souvent. Vous voulez dire quelque chose de neuf et épater le monde entier. Vous parlez comme si vous étiez encore le secr(étaire) gén(éral) du Kom(intern) et que vous donniez un interview à un journal com(muniste). Vous fournissez les arguments aux élém(ents) réact(ionnaires) en Amér(ique) pour convaincre l'opinion publ(ique) que l'Amér(ique) ne fera rien d'extraordinaire si elle crée un bloc occid(ental), puisque dans les Balk(ans) il y a non seulement un bloc, mais même une union douan(ière). À présent, en Amér(ique), on mène une vaste campagne élect(orale). Quel gouv(erne)ment il y aura là-bas est pour nous d'une grande importance. Car l'Amérique est un État puissant, bien armé. À la tête de son gouv(erne)ment se trouvent des gens instruits, de vrais sacs d'argent, qui nous détestent furieusement et ne cherchent que des prétextes pour nous faire tort. Ils peuvent échouer aux élections, si nous fournissons, par notre comportement, des 
arguments aux éléments progres(sistes). Mais si nous fournissons des arguments aux réactionnaires, nous les aiderons. Le gouv(erne)ment amér(icain) est à la veille d'élections et en craint les conséquences. Mais si dans le gouv(erne)ment se retrouvent à nouveau ces magnats de la finance, nous en serons responsables dans une mesure signif(icative) par notre comportement. Ils diront: «vous ne créez pas seulement un bloc, mais vous réunissez toute une série d'États : contre qui ?».

Quel besoin avez-vous d'un tel bloc? En fin de compte, si vous voulez vous réunir, pourquoi faire tant de bruit à ce propos? Ou bien vous êtes des ingr(a)ts, ou bien vous vous emportez comme des komsomols, qui se jettent comme des papillons droit vers la flamme.

Quel besoin avez-vous de tout cela ? Pourquoi facilitez-vous la position de vos ennemis en Angl(eterre), en Amér(ique) et en France?

Ou bien, à propos de l'Albanie, comme la question se résoud facilement par les camarades yougosl(aves)! À l'époque de la guerre, les trois États alliés ont stipulé l'indépend(ance) de l'Alb(anie) et déclaré qu'ils appuieront cette indépendance. Dans toutes les hypoth(èses) de lutte entre la rédact(ion) et la démocr(atie), le maillon alb(anais) est notre point le plus fragile. L'Albanie n'a pas encore été admise à l'ONU ${ }^{15}$, les Anglais et les Américains ne la reconnaissent pas. La question là-bas reste ouverte. Il n'y a pas d'autre point aussi fragile. Seule l'Alb(anie) n'a pas de protection jurid(ique) dans les rel(ations) intern(ationales). Si Tito y fait rentrer une division, ou même seulement un régiment, cela n'échappera pas à l'attention des Am(éricains) et des Ang(lais). Ils commenceront à clamer que l'Alb(anie) est occupée. Est-ce que l'Alb(anie) s'est adressée publiqu(ement) à la Yougosl(avie) pour de l'aide ? Et alors ces salopards en Angl(eterre) et en Am(érique) vont se mettre en position de défenseurs de l'indépendance alb(anaise). Qui, sinon un nigaud, va entreprendre de construire un front, qui ne présente, à l'évidence, aucune perspective? À présent, il faut activer l'organ(isation) de cette armée. Qu'on lui donne des instructions, de l'armement. Après quoi, si l'Alb(anie) est attaquée, elle devra appeler à l'aide la Yougosl(avie). Sinon, la Yougosl(avie) apparaîtra comme un pays qui occupe un autre État indépendant. Et alors une intervention étrangère est tout à fait possible. Il y a des bases, des navires américains. Ce serait la position la plus avantag(euse) et commode pour les Amér(icains). Quand on part en guerre, on doit organiser son front de la manière la plus avantageuse, tandis qu'ici on expose carrément ses arrières pour se faire battre par les Amér(icains).

Voyez la guerre qui se mène en Chine. Il n'y a pas un seul de nos soldats là-bas. Est-ce que les Al(ba)nais valent moins que les Chinois? Entraînez-les, armez-les et ils sauront se défendre tout seuls. Il vaut bien mieux qu'ils défendent eux-mêmes leur indépendance.

Les Améric(ains) pourraient difficilement prendre l'initiative d'attaquer, mais autrement leur tâche est facilitée. Vous décidez de ces questions bien facilement, alors qu'elles sont complexes.

Si les partisans grecs sont battus, entrerez-vous en guerre?

$\mathrm{K}(\operatorname{arde}) 1(\mathrm{j})$ : Non.

$53 \mathrm{~S}$ : Je pars de l'analyse des forces en présence des partis(ans) et de leurs adversaires. Ces derniers temps, je commence à douter de la victoire des partis(ans). Si vous n'êtes pas convaincus que les partis(ans) vont l'emporter, il faut replier ${ }^{16}$ le mouv(ement) 
partis(an). Les Amér(icains) et les Angl(ais) s'intér(essent) énormément à la mer Médit(erranée). Ils veulent avoir une base en Grèce et n'épargneront pas leurs moyens pour maintenir là-bas un gouv(erne)ment qui leur soit obéissant. C'est une question internat(ionale) import(ante). Si on replie le mouv(ement) part(isan), ils n'auront pas de prétexte pour vous attaquer. Ce n'est pas si facile que ça que de commencer une guerre maintenant. Si vous êtes convaincus que les partis(ans) ont des chances de l'emporter, alors c'est une autre question. Mais j'ai quelques doutes à ce sujet. G D : Nous recevons peu d'informations d'ici, aussi.

S : Vous avez le droit de nous réclamer que nous vous tenions au courant. Établissons alors un protocole de consultation obligatoire entre nous pour toutes les questions internationales importantes.

D : Nous considérerons ce point.

$\mathrm{S}$ : Le traité avec la Bulg(arie). Nous ne tarderons pas beaucoup ${ }^{17}$. Le 15 les Hongrois seront ici. Après cela, nous nous tournerons vers [illisible], puis ensuite vers vous.

M : Dans un des traités, on mentionne l'anéantissement de tous les foyers d'agression. Ce ne sont que de belles phrases qui ne font que donner des armes inutiles à vos ennemis. Pourquoi déclarer davantage que ce qu'il y a dans les autres traités ? Est-ce une guerre préventive que vous voulez mener?

$\mathrm{S}:$ Ce sont des dérapages gauchistes.

M : Par le traité, on appuie toute initiat(ive) de l'ONU contre les agresseurs. Ils peuvent s'arranger pour que cela se retourne contre nous. La [illisible] des nations ne nous a-t-il pas déclaré agresseurs à propos de l'aff(aire) de Finlande?

Quant aux camarades yougosl(aves), ils n'ont pas autorisé les Albanais à acheter chez nous 5000 tonnes d'avoine et les ont envoyé chercher de l'avoine en Argentine.

S: Les Yougoslaves ont visiblement peur que nous ne leur enlevions l'Albanie. Vous devez prendre l'Albanie, mais de façon intelligente. Quant [illisible] tr(aité) avec la Roumanie est en harmonie avec Moscou.

Avec l'arrêté d'harmonisation des plans écon(omiques), vous réduisez votre souveraineté et celle de la Roumanie.

Il n'y a que trois fédérations qui soient possibles et naturelles : 1/ Yougosl(avie) et Bulg(arie) ; 2/ Roum(anie) et Hong(rie) ; 3/ Pologne et Tchécosl(ovaquie). Voilà, de telles fédérations sont possibles et réalistes. Une confédération entre elles est une élucubration.

$\operatorname{Tr}$ (ajčo Kostov) : Doit-on considérer que nous pouvons adopter une ligne d'accélération de la fédér(ation) entre la B(ulgarie) et la Yougosl(avie) ?

S : Faites-le, si vous le voulez, dès demain. C'est tout naturel et nous n'avons rien contre. Nous sommes contre les méthodes de komsom(ols) dans les fédérations. Une fédér(ation) doit être préparée, afin d'être acceptable par les ennemis à l'intérieur du pays et dans le monde extérieur.

$\mathrm{K}(\operatorname{arde}) l(\mathrm{j})$ : Nous pensons que, même avec la B(ulgarie), il ne faut pas se hâter, surtout pour des motifs internat(ionaux), car cela peut compliquer notre situation.

S : Ici, vous vous trompez. Il ne faut pas que vous tardiez dans l'unific(ation) des trois pays Yougosl(avie), Bulg(arie) et Alb(anie). Mais il est indispensable que les assemblées nat(ionales) en prennent la décision et donnent l'ordre aux gouv(erne)ments de 
commencer des pourparlers d'unification. Mieux vaut commencer par l'unification polit(ique) et ensuite envoyer des troupes en Albanie. Alors cela ne pourra plus servir de prétexte à représailles.

Il était prémat(uré) de faire une fédér(ation), tant que la paix n'était pas établie en Bulg(arie). Mais à présent, la Bulg(arie) est un État normal et de plein droit. À mon avis, maintenant il ne faut pas retarder cette question; mieux vaut l'accélérer. Si vous accélérez l'union par les assemblées nat(ionales), tout ira bien. La fédération résoud tous les problèmes. Entre Bulgares et Yougoslaves, la proximité sur le plan racial et sur le $\mathrm{pl}(\mathrm{an}) \mathrm{du}$ mode de vie est très grande et chacun comprendra cette unification. Les Albanais gagnent aussi à une fédération parce qu'une Albanie unifiée sera créé, avec une pop(ulation) presque doublée.

70 Il faut concentr(er) tous les efforts autour d'une fédération naturelle, développez son économie, sa culture nationale, renforcez les armées. Par ailleurs, la Pologne ne met pas ses espoirs en vous ; vous et la Pologne, il n'en sortira rien, il n'y a aucun besoin de nous éloigner des déc(isions) des 9 partis com(munistes).

71 M: S'il est nécessaire d'anéantir les repaires d'agression dont parle le cam(arade) Dim(itrov), faites-le, mais quel besoin y a-t-il de le crier alentour. À présent, les gens sont à l'affût du moindre mot.

$72 \operatorname{Tr}(a j c ̌ c o$ Kostov) : Nous considérons qu'une déf(aite) du mouv(ement) part(isan) en Gr(èce) créerait une situat(ion) très difficile pour les autr(es) pays balk(aniques).

$\mathrm{S}$ : Évidemment, les part(isans) doiv(ent) êt(re) sout(enus). Mais si les persp(ectives) du mouv(ement) part(isan) sont en baisse, mieux vaut repousser le comb(at) à des tem(ps) meilleurs. Ce qui passe pour un rapport de forces ne peut être compensé par des imprécations et [illisible, VB]. Un bilan raisonnable des forces est indispensable. S'il montre qu'à un moment donné, ça ne marche pas, il ne f(aut) pas que nous hésit(ions) à le recon(naître). Il est déjà arrivé que des mouv(ements) de part(isans) se soient repliés, quand les circ(onstances) étaient défavorables. Si aujourd'hui ce n'est pas possible, ce le sera demain.

Vous avez peur de poser le problème brutalement. Vous vous laissez impres(sionner) par la "dette morale". Si vous ne parvenez pas à soulever un poids donné, dont vous vous êtes chargés, il $\mathrm{f}(\mathrm{aut})$ le recon(naitre). Il ne faut pas que vous redoutiez un quelconque "impératif catégorique" sur la question de la dette mor(ale). De tels impér(atifs) catég(oriques) n'existent pas chez nous. Toute la question réside dans l'équil(ibre) des objectifs ${ }^{18}$. Si tu es bien, frappe. Sinon, refuse le combat. Nous accept(ons) le combat, non pas quand le veut l'advers(aire), mais quand c'est dans notre intér(êt).

$\mathrm{K}($ ardel)j : La quest(ion) des chanc(es) des partis(ans) se clarifiera d'ici quelques moi(s).

$\mathrm{S}$ : Bon, dans ce cas, attendez. Vous avez peut-être raison.

Moi aussi j'ai douté que les Chinois réussissent et je leur ai conseillé de s'entendre provisoirement avec Tchang Kaï-chek. Formellement, ils ont été d'accord avec nous, mais en pratique, ils ont continué leur idée : mobiliser les forces du peuple chin(ois).

Après quoi ils ont posé ouvertement la question : nous continuerons à nous battre, le peuple nous soutient. Nous avons dit : bien, de quoi avez-vous besoin? Il s'est avéré que chez eux, le terreau est très favorable. En fin de compte, ils avaient raison et nous tort. 
Peut-être qu'ici aussi nous aurons tort en fin de compte. Mais nous voulons agir à coup sûr.

\section{l'harmonisation des plans, c'est parce que nous avons besoin de nous aider, d'accélérer} notre développement écon(omique). vous pouvez fusionner. Mais je ne vois pas en quoi pourrait vous aider une union douan(ière) et l'harmonisation des $\mathrm{pl}$ (ans) avec la Roum(anie). Les Roum(ains) vendent leurs produits bon mar(ché) ou plus avantageusement.

$\mathrm{K}($ olarov) V(asil) : Les Américains toléreront-ils une victoire des partis(ans) ? hommes qui peuvent utiliser les forces pop(ulaires), il f(aut) continuer le combat. Mais il ne f(aut) pas penser que si cela n'aboutit pas en Grèce, tout est perdu.

Les États limitr(ophes) doiv(ent) reconnaître le gouv(ernement) de Markos en dernier. Que les autres le reconnaissent d'abord, comme plus éloignés ${ }^{19}$.

M : La déclar(ation) du cam(arade) Dim(itrov) contre l'opposition de Lulčev ${ }^{20}$ était aussi une erreur, qui est utilisée maintenant si largement par les ennemis. Quel besoin de cela?

D : C'était surtout provoqué par un besoin intér(ieur), pour qu'ils n'essaient pas de relever la tête à nouveau.

S : Que Jugov discute avec l'opposit(ion), il sait bien le faire ${ }^{21}$.

$\mathrm{K}(\operatorname{ardel}) \mathrm{j}$ : Nous considérons qu'il n'y a pas de diverg(ences) de fond entre nous. Il s'agit d'erreurs ponct(uelles).

$\mathrm{S}$ : Ce ne sont pas des erreurs ponct(uelles), mais un système.

Dans le traité avec la Tchécosl(ovaquie), vous pouviez vous contenter de la formule tchèque : si l'All(emagne) ou une puissance en alliance avec elle attaque, s'il y a danger d'attaque de la part d'un autre pays, alors entre en vigueur la clause sur la consultation mutuelle ${ }^{22}$

L'aide à l'émigration en Palestine des juifs passe selon les dir(ectives) de l'ONU pour l'installation en Palestine de 400000 p(ersonnes). L'ONU a fait preuve de bienveil(lance) envers les revendications j(uives). Comme ça, les Ang(lais) n'ont aucun droit de protester.

Molotov : divergences de vues sur trois questions :

1/ le traité bulg(aro)-yougosl(ave); 2/ interview de G D; 3/ entr(ée) de l'armée yougosl(ave) en Alb(anie).

93 L'erreur était de conclure le traité b(ulgaro)-youg(oslave) avant l'entrée en vig(ueur) du tr(aité) de p(aix) et de façon illimitée, sans consultation avec l'U(nion) Sov(iétique).

Cette précipitat(ion) a renforcé les posit(ions) des élém(ents) réact(ionnaires) en An(gleterre) et en Am(érique), qui y ont trouvé prétexte à renforcer l'interv(ention) en Grèce.

Le gouv(ernement) sov(iétique) avert(it) qu'il ne peut prendre la responsab(ilité) de pactes d'une importance aussi énorme, surt(out) sans consult(ation) de l'URSS. 

yougosl(ave) en Alb(anie) pour la déf(ense) conj(ointe) de la frontière. Les Alb(anais) ont cru que c'était avec l'acc(ord) de $\mathrm{M}$ (oscou). Après notre intervention, cela a été ajourné. De même, les Yougosl(aves) ont envoyé les Alb(anais) ach(eter) de l'avoine en Arg(entine).

De telles relat(ions) sont incorr(ectes) et inadmissibles.

II

Le cam(arade) G. D. s'est laissé emporter dans la conf(érence) de presse et les interviews, et fournit l'occasion d'être provoqué, à parler d'affaires dont il ne convient pas de parler.

Dans l'interview est exposé un plan de grande portée sans la moin(dre) tentative d'harmonisation avec qui que ce soit.

C'est incorr(ect) dans le principe et tactiquement nuisible.

Par cela vous facilitez la tâch(e) de la création d'un Bloc occidental, puisque la réaction considère que c'est sur l'instig(ation) de l'URSS. À ce sujet, G. D. a reçu un avertiss(ement) selon la ligne du part(i).

En fait :

La création d'une fédér(ation) ou confédér(ation) inclu(ant) la Pologne, la Tchécosl(ovaquie), la Grèce est irréaliste et irréalisable. Trois fédérations sont réalistes: $\mathrm{B}($ ulgarie) et Yougosl(avie), Roum(anie) et Hong(rie), Pologne et Tchécosl(ovaquie).

La même chose concernant l'union douan(ière). Nous avons bien établi que le projet de tr(aité) avec la $\mathrm{R}$ (oumanie), qui comprend une clause sur le non-rec(ours) à l'union douan(ière) a été mis en accord et approuvé à $\mathrm{M}$ (oscou). Après vérification, ils ont dit que c'était exact, qu'ils «s'en étaient rendu compte ». Mais que, foncièrement, l'union douan(ière) avec la Roum(anie) était aussi "irréaliste".

Les cam(arades) polonais ont cru que l'idée de féd(ération) venait de Moscou, mais quand ils ont su que ce n'était pas vrai, ils ont dit qu'ils n'étaient pas d'accord non plus. L'organe de Beneš a également rejeté la resp(onsabilité) de ce "pataquès" sur les com(munistes) tchèques.

La position de G. D. est en contradict(ion) avec la décl(aration) des 9 (partis communistes).

Les éclaircis(sements) de G. D. n'ont aidé en rien.

Ce ne sont pas les intent(ions) qui comptent, il faut aussi penser aux concl(usions) qu'en tireront les autres.

Complémentairement, St(aline) a précisé que la féd(ération) entre la Youg(oslavie) et la $\mathrm{B}$ (ulgarie) pouvait se réal(iser) dès demain, mais qu'il fal(lait) le faire avec sagesse.

III

Diverg(ences) dans l'appréciation de la sit(uation) internat(ionale).

Il est indispensable de se consulter sur les questions internat(ionales) import(antes). Un protocole particulier à ce sujet. 
St(aline) : 1/ Il y a entre nous des divergences importantes, et pas seulement des erreurs. Il ne faut pas le nier et le cacher. Il n'y a là rien de dangereux. Chez nous aussi, il y a eu des diverg(ences), même avec Lén(ine).

2/ Des interv(iews) de ce genre alimentent la réact(ion) amér(icaine) pour convain(cre) l'opinion publ(ique) en faveur d'un Bloc occidental. L'Amérique est à la veille d'élections. Ceux qui la gouv(erneront) demain ne sont pas indif(férents). Elle est gouv(ernée) actuellement par des sacs d'arg(ent). Citation 1 (8-10).

[Ici Kolarov cesse de rapporter exactement la conclusion de Staline, car il répète en bref ses arguments. Les chiffres renvoient aux pages où l'argumentation est présentée en détail dans les notes.]

3/ Albanie. Citation 2 (11-14).

4/ Partisans grecs. Citation 3 (15-16, 24-28).

5/ Traité avec la Bulgarie.

Le 15 février avec les Hong(rois), après ça, ils se tournent vers les Finnois (?). Après cela la Bulgarie (17).

6/ Anéantissement des foyers d'agression. Citation 4 (17-18, 23).

7/ Pour la fédération. Citation 5 (19-23).

8/ L'opposition de Lulčev. Citation 6 (M(olotov) 29).

9/ Comment doit se présenter le projet de pacte avec la Tchécosl(ovaquie). Citation 7 (30).

10/ Aider les juifs à émigrer en Palestine. Citation 8 (30).

11/ Pourparlers avec la Turquie. Accord.

\section{Conclusions}

1/ On admet la critique légitime adressée par le cam(arade) Staline et le cam(arade) Molotov à propos du traité bulgaro-yougoslave et de l'interview du cam(arade) G. Dimitrov accordé aux journalistes dans le wagon entre Bucarest et Giurgiu et on prend acte des recommandations qu'ils ont données et on les approuve.

On admet qu'en l'occurrence, il ne s'agit pas de fautes ponctuelles, mais d'une différence de vue significative dans l'appréciation de la situation internat(ionale) en général, et dans les Balkans et en Bulgarie en particulier.

La précipitation à conclure le traité bulgaro-yougoslave sans consultation de l'URSS, le plan annoncé publiquement d'une large fédération ou confédération, qui engloberait même la Tchécoslovaquie, la Pologne et la Grèce, sans que cela ait été discuté et décidé par les pays concernés et approuvé par l'URSS, la déclaration sur l'anéantissement de tous les foyers d'agression, l'idée d'une large union douanière, dans l'annonce de laquelle on pouvait comprendre que cette union engloberait aussi l'URSS - tout cela est fondamentalement incorrect, irréalisable et politiquement nuisible, car cela facilite les menées des réactionnaires en Amérique et en Angleterre pour abuser l'opinion publ(ique) et la gagner à l'idée d'un bloc occidental, dirigé contre les nouvelles démocraties et l'URSS.

2/ On approuve le protocole signé de consultation mutuelle entre la Bulgarie et l'URSS sur toutes les questions internat(ionales) importantes et l'on fait obligation aux 
camarades dirigeants respectifs de respecter exactement et honnêtement cet engagement.

3/ On admet que la situation en Grèce doit être soumise à une évaluation sévère (et) objective ${ }^{23}$, en partant d'une appréciation correcte du rapport de forces [la mention « et non en raison d'une dette morale " a été raturée dans le texte]. De toute façon, il faut fournir de l'aide au peuple grec par des actes, sans faire de bruit autour.

4/ On admet que seule est réaliste et réalisable pour nous la fédération entre la Bulgarie et la Yougoslavie (et l'Albanie) et l'on est d'accord pour procéder à sa réalisation dans les plus brefs délais, l'initiative devant en émaner des assemblées nationales des deux pays. Il est indispensable d'entrer sans retard en contact avec le CC du PCY, pour discuter conjointement de la question.

5/ On approuve d'entreprendre des ouvertures tactiques d'amélioration des relations entre la Bulgarie et la Turquie.

6/ On décide d'organiser un contrôle sur la presse pour les questions internationales.

\section{NOTES}

1. Djilas (Milovan), Conversations avec Staline, Paris : Gallimard, 1962, pp. 189-204.

2. Mičev (Dobrin), Makedonskiat văpros v Bălgaro-jugoslavskite otnošenija 9-IX-1944 - 1949 (La question macédonienne dans les relations bulgaro-yougoslaves), Sofia, 1994, pp. 385-397.

3. Angelov (Veselin), «Edin dokument za sădăržanieto i rezultatite ot săvetsko-jugoslavkobălgărskata tajna srešta v Moskva na 10.II.1948 » (Un document sur la teneur et les résultats de la réunion secrète soviéto-yougoslavo-bulgare à Moscou le 10/02/48), Istoričeski pregled, 44 (1-2), 1998.

4. Rabotničesko Delo, (14), 20 janvier 1948, cité par ibid., p. 229.

5. Ibid.

6. Petranović (Branko), Zečević (Momčilo), Jugoslavija 1918-1984. Zbirka dokumenata (Yougoslavie, 1918-1984. Recueil de documents), Beograd: Rad, 1985, p. 745 (tout comme M. Djilas, ils indiquent que l'article de la Pravda date du 29 janvier).

7. Angelov (Veselin), art.cit., p. 230.

8. À comprendre dans le contexte de la famille balkanique traditionnelle où les enfants mariés vivent sous le même toit que leurs parents. La belle-mère ne veut pas réprimander directement la “pièce rapportée" qu'est sa bru, et adresse ses reproches à sa fille qui n'y est pour rien !

9. Quant aux notes, elles sont toutes de Bernard Lory.

10. Un traité bulgaro-serbe a été signé le 31 mars 1904. Ses clauses secrètes prévoyaient un statu-quo territorial en Turquie d'Europe. Une union douanière entre les deux pays est signée le 9 juillet 1905, mais l'intervention de l'Autriche-Hongrie empêchera sa mise en application.

11. Le Traité de Paris, signé le 10 février 1947, entrait en vigueur le 15 sepembre. Les accords bulgaro-yougoslaves de Bled datent du $1^{\mathrm{er}}$ août.

12. Le "coup de Prague" eut lieu moins de deux semaines plus tard (20-25 février 1948).

13. Le texte de la déclaration des 9 partis communistes, daté sans plus de précision, de septembre 1947 est fourni par Petranović (Branko), Zečević (Momčilo), op.cit., pp. 740-742. C'est lors de cette rencontre que le Kominform a été créé. 
14. Erreur, il faut lire S, évidemment.

15. Elle ne le sera que le 14 décembre 1955.

16. Djilas note que Staline utilise une expression populaire svernut, dans le sens de "fumer de la barague", Djilas (Milovan), op. cit., p. 199.

17. Le traité de coopération soviéto-bulgare (pour une durée de 20 ans) fut signé le 18 mars 1948.

18. Probable erreur de lecture : sili (forces) serait plus logique que celi (objectifs).

19. Le gouvernement démocratique provisoire grec a été créé le 24 décembre 1947. Il ne sera reconnu officiellement par aucun pays de l'est.

20. Kosta Lulčev, leader de la fraction du Parti social-démocrate, en opposition au Front de la Patrie.

21. Anton Jugov, ministre de l'Intérieur.

22. Le traité d'amitié bulgaro-tchécoslovaque sera signé le 23 avril 1948.

23. On peut aussi comprendre strictement objective. 